\title{
In Memoriam Wolfgang Boehm
}

Professor Wolfgang Boehm passed away on May 1, 2018, 11 days short of being 90 years old.

He was an early pioneer in the application of splines to Computer-Aided Geometric Design (CAGD), which provides the mathematical basis for the use of computers to design, engineer and manufacture products and complex systems.

My first encounter with Prof. Boehm's work was in early 1981. I was new to CAGD and particularly interested in developing rational B-spline technology for use in engineering applications. Upon discovering his 1980 paper: "Inserting new knots into B-spline curves," my first impression was: what a beautiful work. It was short, just three pages, elegantly simple and clear, but eminently useful. The applications were immediately clear: curve/surface division, modification by means of control point refinement, decomposition into Bezier and other polynomial forms, and rendering sets of curves compatible for the purpose of surface constructions such as lofting, just to name a few. This paper had a profound influence on my work.
Prof. Boehm authored many papers during the 1980s. At the right time in a relatively new field of mathematical research, a good survey paper can be quite valuable. His 1984 paper: "A survey of curve and surface methods in CAGD" is an excellent example. At the other end of the length spectrum, 60 pages, it reflected his presentation style: artistically illustrated, well organized, clearly explained, and useful.

Prof. Boehm was the founder of the Geometric Modeling Lab at the Technical University at Braunschweig, Germany. Over his many years, he mentored many students and researchers, some of whom went on to make important contributions to CAGD. A global leader in CAGD, he was the founder and first Editor-in-Chief of the journal: Computer-Aided Geometric Design. He was in attendance at many conferences, often as an organizer, but always as an interesting and much anticipated contributor. He leaves a large footprint in the CAGD community.

Wayne Tiller Co-author of The NURBS Book @waynetiller2@gmail.com 